\title{
MULTIPLE PRIMARY MALIGNANT NEOPLASMS- AN INTERESTING CASE REPORT
}

\author{
Chandni Krishnani ${ }^{1}$, R. K. Tandon², S. S. Goswami3 ${ }^{3}$ Payal Desai ${ }^{4}$
}

${ }_{1}^{1}$ Resident, Department of Pathology, SBK Shah MI and RI, Vadodara, Gujarat, India. 2Professor, Department of Pathology, SBK Shah MI and RI, Vadodara, Gujarat, India. 3 Professor, Department of Pathology, SBK Shah MI and RI, Vadodara, Gujarat, India. ${ }_{4}^{4}$ Resident, Department of Pathology, SBK Shah MI and RI, Vadodara, Gujarat, India.

HOW TO CITE THIS ARTICLE: Krishnani C, Tandon RK, Goswami SS, et al. Multiple primary malignant neoplasms- an interesting case report. J. Evolution Med. Dent. Sci. 2018;7(39):4318-4320, DOI: 10.14260/jemds/2018/962

\section{PRESENTATION OF CASE}

A 65-year-old male with complaints of lump in left breast since 2 years. CT scan findings were non-homogeneous mass in left breast along with bilateral axillary lymphadenopathy. Lumpectomy was performed and diagnosed as infiltrating duct carcinoma breast. After that, we received modified radical mastectomy specimen in our lab. On gross examination, subareolar cavity was surrounded by whitish solid area of $2 \mathrm{~cm}$ without involvement of skin. Total 26 lymph nodes were retrieved. Lymph nodes were whitish and fleshy \{Fig. 1\}. The resection specimen was fixed in formalin, routinely processed and multiple representatives of 3-micron sections were stained with haematoxylin and eosin stain and Immunohistochemistry (IHC) was also performed. Microscopic examination showed the features of infiltrating duct carcinoma NOS: Modified Nottingham Bloom Richardson's Grade II in breast specimen. All the lymph nodes were submitted for processing and $\mathrm{H}$ and $\mathrm{E}$ stained sections taken from all the submitted lymph nodes showed the complete effacement of lymph node by small lymphocytes having slightly irregular round nuclei. The chromatin was condensed, and cytoplasm was scanty [Fig. 3]. None of the lymph nodes showed any metastatic deposits. Peripheral smear of patient showed features of chronic lymphocytic leukaemia (absolute lymphocyte count was 16400 cells $/ \mathrm{mm}^{3}$ ] [Fig. 5].

IHC of breast tumour showed ER/ PR positivity with $\mathrm{H}$ score of 350 and 240 respectively and HER-2/ Neu protein expression was negative with score of 1+ [Fig. 2]. Lymph nodes were immunoreactive for CD 19, CD 23 and for CD 5. Cells were non-reactive for Cyclin D1 a and CD3 [Fig. 4].

\section{FINAL DIAGNOSIS}

IDC breast Stage II B with Small lymphocytic lymphoma and chronic lymphocytic leukaemia.

'Financial or Other Competing Interest': None.

Submission 13-08-2018, Peer Review 12-09-2018,

Acceptance 18-09-2018, Published 24-09-2018.

Corresponding Author:

Dr. Chandni Krishnani,

L406,

Samsara Luxury Apartments,

Chhani Road,

Vadodara-390024,

Gujarat,

India.

E-mail: itschandnik@gmail.com

DOI: $10.14260 /$ jemds $/ 2018 / 962$
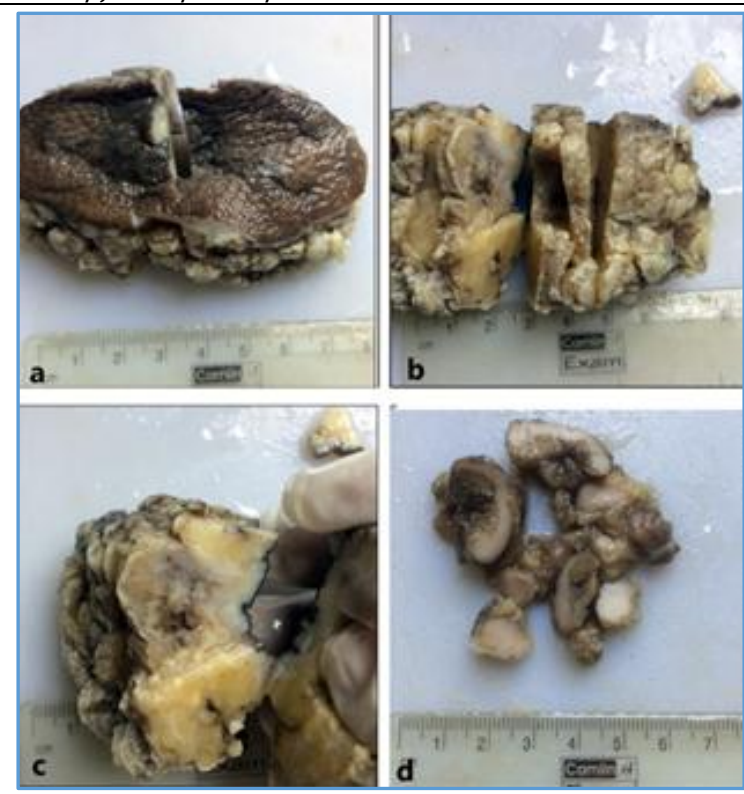

Figure 1. Gross Specimen of Post Lumpectomy MRM Specimen

a. Superior Surface-Skin not Involved b. Cut Section Breast

c. Post Lumpectomy Cavity with surrounding grey white Tumour Area.

d. Gross Specimen of Lymph Node showing Large, Whitish, Fleshy Lymph Nodes

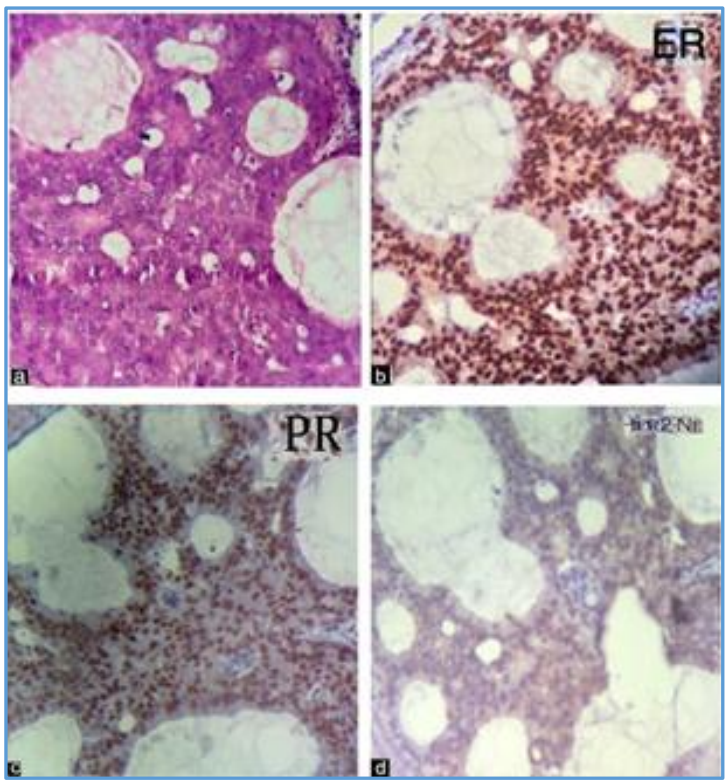

Figure 2. Pathology and Immunohistochemistry a. Invasive Ductal Carcinoma, NOS Breast (40x)

b. Immunostaining for ER: + (H score 350$)$

c. Immunostaining for PR: + (H score 250)

d. Immunostaining for HER-2: Negative 


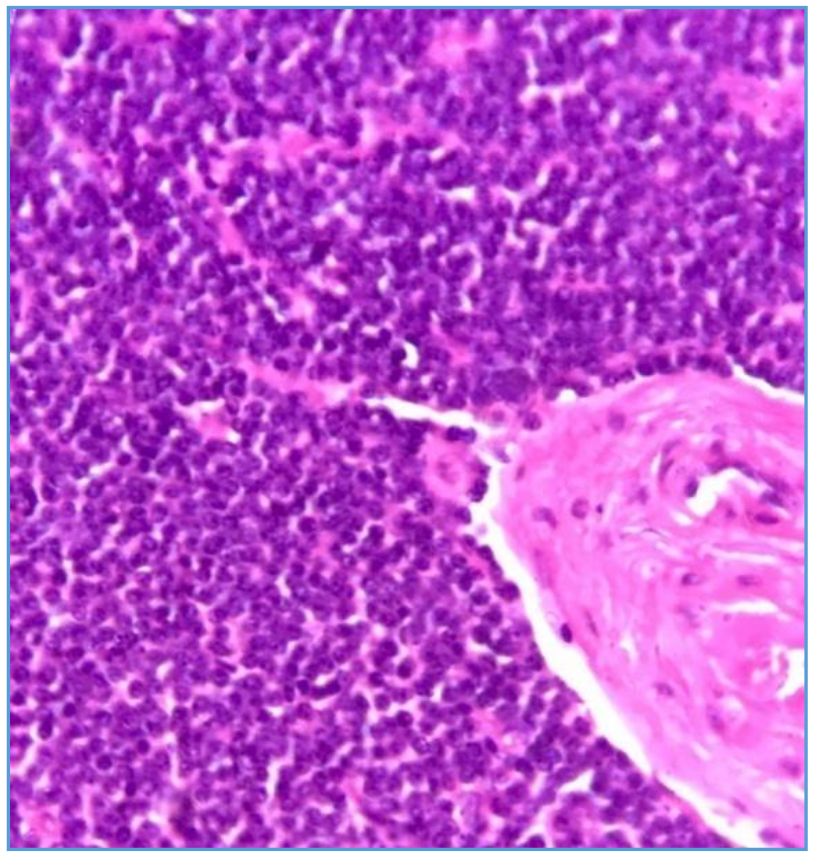

Figure 3. Axillary Lymph Node-Small Lymphocytic Lymphoma (40x) (H and E)

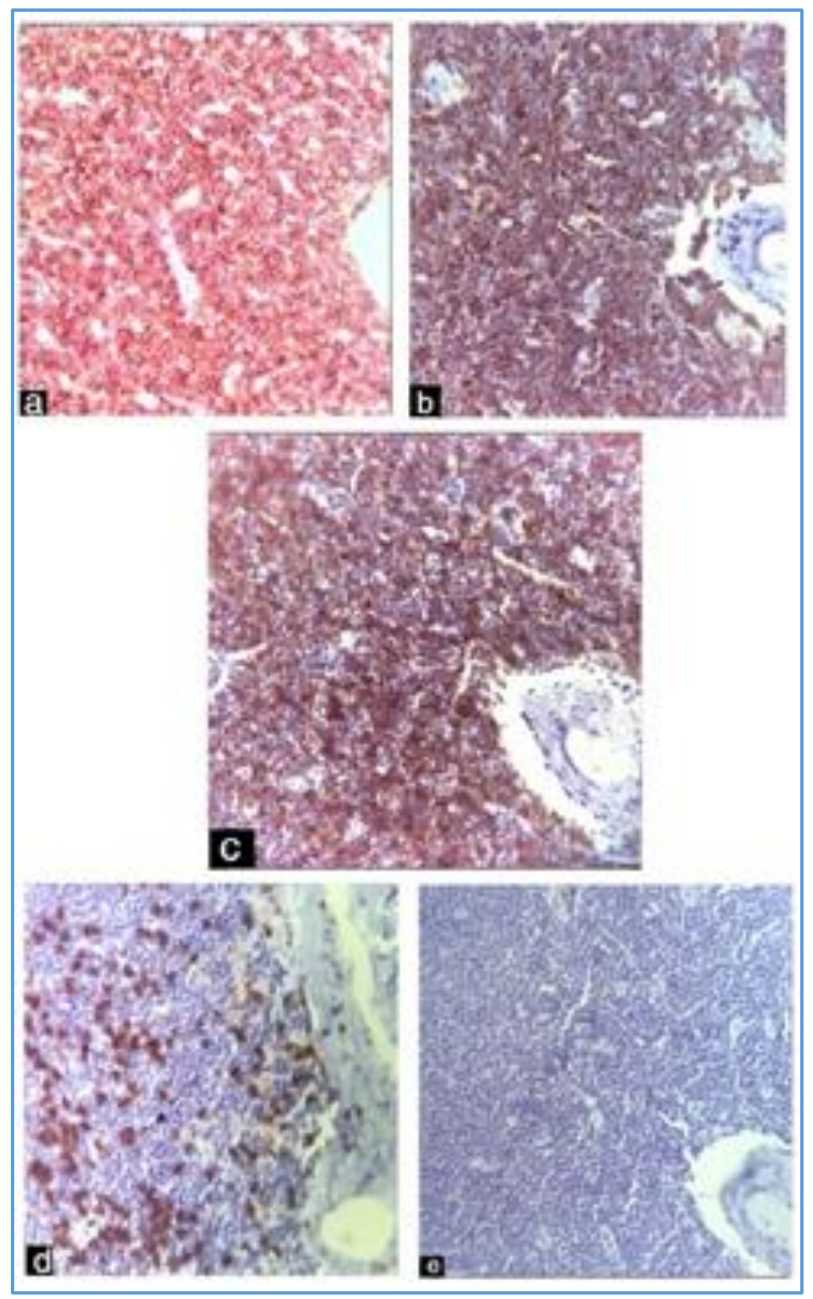

Figure 4. Immunohistochemistry Lymph Node

a. CD20 Strongly Expressed
b. CD23 Strongly Expressed
c. CD5 Strongly Expressed

c. CD5 Strongly Express
d. CD3 Not Expressed

e. Cyclin D 1a. Not Expressed

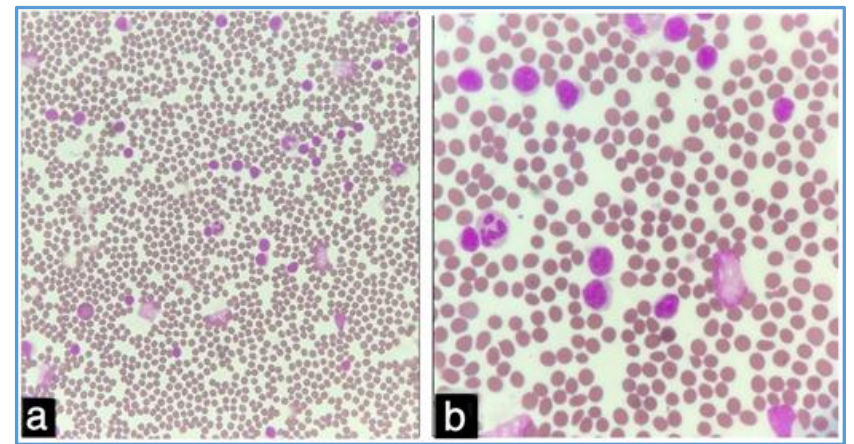

Figure 5. Peripheral Smear showing Mature Lymphocytes and Smudge Cells (ALC= 19400 cells $/ \mathrm{mm}^{3}$ )
a. 40x
b. 100x

\section{PATHOLOGIC DISCUSSION}

Multiple Primary Malignant Neoplasms (MPMNs) are rarely reported and are defined as the diagnosis of $\geq 2$ independent, primary malignancies of different histologies/ origins in a single individual. The prevalence of MPMNs varies from $0.73 \%$ to $11.70 \%$ of all patients diagnosed with carcinomas in western countries.[1] In this study, we report a patient with Male Breast Cancer (MBC) and coexisting Small Lymphocytic Lymphoma (SLL) and Chronic Lymphocytic Leukaemia (CLL).

In the United States only $1 \%$ of all breast carcinomas occur in males, but in some Arab countries the incidence rises to nearly $10 \% .{ }^{[2-4]}$ Clinically, most breast carcinomas present in elderly men as breast masses with or without associated nipple abnormalities.[5] Similarly, grossly, microscopically and immunohistochemically, carcinomas of the male breast are like those seen in females. As such they can be in situ or invasive, low grade or high grade. All of the microscopic types and molecular subtypes identified in the female breast have been encountered in males.

Incidental lymphomas are discovered in a small percentage of patients who undergo radical lymph node dissection for staging of various carcinomas. The most common type of lymphoma found in these patients is CLL/SLL.[6]

The diagnosis of multiple primary malignancies in this study was made according to the criteria developed by Warren and Gates.[1] Each tumour must be malignant, each tumour must have its own unique pathological features and metastasis or recurrence must be excluded. Depending on the time of diagnosis, the dual malignancies can be synchronous or metachronous. This case showed a combination of MBC and SLL and CLL, two completely different cancer types diagnosed at the same time and therefore met the criteria for simultaneous MPMNs.

The pathogenesis behind increased tendency to develop multiple tumours (Synchronous or Metachronous) in some subjects probably may be either due to an individual predisposition or by the action of carcinogenic factors acting on different organs at different times. The pathogenesis for development of whether single or multiple tumours have similar mechanisms. The combined action of various genetic and environment factors initiate and propagate the onset of a new tumour. Therefore, for the development of MPMN multiple factors and predisposing factors must be acting.[7]

The frequency of $\mathrm{MBC}$ is $1 \%$ in Western nations, $0.5 \%$ in some piece of Asian countries.[8] Review of reconnaissance, 
the study of disease transmission and final product (SEER) information show an ascent in the rate of $\mathrm{MBC}$ from $1.0 / 100,000$ men in the late 1970 s to $1.2 / 100,000$ men from 2000 to 2004.[9] There is absence of information on male breast malignancy from India. Risk factors for the development of male as well as female breast cancer are similar and include increasing age, first degree relatives having carcinoma breast, exogenous oestrogen therapy and ionising radiations. Other factors are obesity, infertility and pre-existing proliferative benign breast disease. From 3\% to $5 \%$ of cases are associated with Klinefelter syndrome and decreased testicular functions. The typical age at diagnosis is between 60 and 70 years. BRCA 2 germline mutations have been attributed in $4-14 \%$ cases of MBC. MBC is also observed in BRCA 1 families, though not as frequently.[10]

The difference between CLL and SLL is only in the degree of lymphocytosis in the peripheral smear. In most of the affected patients, absolute lymphocyte count $>5000$ cells/cumm; criteria for the diagnosis of CLL. In Western world, CLL is the most common leukaemia of adults with 60 years as median age of the diagnosis. Slight male preponderance is noted with the ratio of 2: 1 . SLL accounts for only $4 \%$ of NHLs. In Japan and other Asian countries CLL/SLL is much less common as compared to West. Chromosomal translocations are also less common in CLL/SLL as compared to other lymphoid malignancies. The deletions of $13 q 14.3,11 q$ and $17 p$ and trisomy $12 q$ is the most common genetic abnormality. CLL/SLL have distinctive immunophenotyping. The cells are strongly immunoreactive for pan B-Cell markers CD19 and CD20, and for CD23 and CD5 also.[11]

In the absence of any therapy, simultaneous presence of both malignancies is rare. These findings suggest that there must be some link between CLL and breast cancer.[11] Simultaneous presence of breast cancer and leukaemia have been reported in BRCA2 germline mutation carrying families.[12,13] In SLL/CLL cases, literature data is available regarding tumour suppressor locus at $13 q 12-13$ at the BRCA2 gene. Also deleted regions on chromosome 13q containing BRCA2 or RB genes have been reported in breast cancer patients. This provides a possible link between these two malignant neoplasms through chromosome 13.

Our case of MPMN may be also associated with some underlying genetic abnormality. However, we cannot conclude that MBC and SLL/CLL in our case occurred simultaneously or consecutively in our case.

Still there are many limitations in the literature for MPMN, because most of the data available is through the case report studies. The incidence of metachronous and synchronous tumours might be relatively higher than the reported cases.[7] Therefore, pathologists, radiologists and clinicians have to be aware about different combinations, patterns and clinical presentation of multiple malignant tumours. And all this is required for the successful management of patient, which can be achieved by multidisciplinary approach required in multiple primary malignant synchronous tumours, thereby increasing the life expectancy of patient.

\section{CONCLUSION}

After the long search made on internet, this is probably the first case of MBC with SLL and CLL. The diagnosis is consistent with synchronous MPMNs, which are increasingly reported nowadays. Careful vigilance is therefore really necessary for early diagnosis of such MPMNs. The underlying mechanisms are unknown. Further studies are required to define the risk factors and any underlying genetic and chromosomal anomalies, which will help in the targeted therapies as well as prevention of MPMNs.

\section{Abbreviations \\ MBC : Male Breast Cancer. \\ SLL : Small Lymphocytic Lymphoma. \\ CLL : Chronic Lymphocytic Leukaemia.}

MPMNs : Multiple Primary Malignant Neoplasms.

\section{REFERENCES}

[1] Billroth T. Die allgemeine chirurgische pathologie and therapie. In: Reimer G, edr. 51 Vorlesungen-Ein Handbuch fur Studierende and Artze, 14. Berlin: Auflage 1889.

[2] Fentiman IS, Fourquet A, Hortobagyi GN. Male breast cancer. Lancet 2006;367(9510):595-604.

[3] Gomez-Raposo C, Tevar ZF, Moyano SM, et al. Male breast cancer. Cancer Treat Rev 2010;36(6):451-7.

[4] Donegan WL. Cancer of the breast in men. CA Cancer J Clin 1991;41(6):339-54.

[5] Anderson WF, Jatoi I, Tse J, et al. Male breast cancer: a population-based comparison with female breast cancer. J Clin Oncol 2010;28(2):232-9.

[6] He H, Cheng L, Weiss LM, et al. Clinical outcome of incidental pelvic node malignant B-cell lymphomas discovered at the time of radical prostatectomy. Leuk Lymphoma 2007;48(10):1976-80.

[7] Xu LL, Gu KS. Clinical retrospective analysis of cases with multiple primary malignant neoplasms. Gen Mol Res 2014;13(4):9271-84.

[8] Cutuli B, Le-Nir CC, Serin D, et al. Male breast cancer. Evolution of treatment and prognostic factors. Analysis of 489 cases. Crit Rev Oncol Hematol 2010;73(3):246-54.

[9] Speirs V, Shaaban AM. The rising incidence of male breast cancer. Breast Cancer Res Treat 2009;115(2):429-30.

[10] Robbins and Cotran. Pathologic basis of disease. Vol. 2. South Asia edition, $10^{\text {th }}$ edn. India: RELX India Private Limited., 2016: p. 1066.

[11] Robbins and Cotran. Pathologic basis of disease. Vol. 1. South Asia edition, $10^{\text {th }}$ edn. India: RELX India Private Limited., 2016: p. 593-4.

[12] Goldgar DE, Neuhausen SL, Steele L, et al. A 45-year follow-up of kindred 107 and the search for BRCA2. J Natl Cancer Inst Monogr 1995;17:15-9.

[13] Berman DB, Costalas J, Schultz DC, et al. A common mutation in BRCA2 that predisposes to a variety of cancers is found in both Jewish Ashkenazi and nonJewish individuals. Cancer Res 1996;56(15):3409-14. 